\title{
Optical Monitoring of Tumors in BALB/c Nude Mice Using Optical Coherence Tomography
}

\author{
Hyun-Woo Song ${ }^{1 *}$, Sang-Won Lee ${ }^{1,3}$, Myung-Hwan Jung ${ }^{2}$, Kye Ryung Kim², Seungkyoung Yang, \\ Jeong Won Park ${ }^{1}$, Min-Sook Jeong, ${ }^{1}$, Moon Youn Jung ${ }^{1}$, and Seunghwan Kim ${ }^{1}$

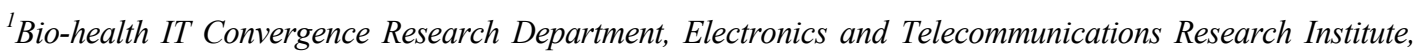 \\ Daejeon 305-700, Korea \\ ${ }^{2}$ Proton Engineering Frontier Project, Korea Atomic Energy Research Institute, \\ Daejeon 305-353, Korea \\ ${ }^{3}$ Division of Convergence Technology, Korea Research Institute of Standards and Science, \\ Daejeon 305-340, Korea
}

(Received October 22, 2012 : revised January 16, 2013 : accepted January 18, 2013)

\begin{abstract}
We report a method for optical monitoring of tumors in an animal model using optical coherence tomography (OCT). In a spectral domain OCT system, a superluminescent diode light source with a full width of $66 \mathrm{~nm}$ at half maximum and peak wavelength of $950 \mathrm{~nm}$ was used to take images having an axial resolution of $6.8 \mu \mathrm{m}$. Cancer cells of PC-3 were cultured and inoculated into the hypodermis of auricle tissues in $\mathrm{BALB} / \mathrm{c}$ nude mice. We observed tumor formation and growth at the injection region of cancer cells in vivo and obtained the images of tumor mass center and sparse circumferences. On the $5^{\text {th }}$ day from an inoculation of cancer cells, histological images of the tumor region using cross-sectional slicing and dye staining of specimens were taken in order to confirm the correlation with the high resolution OCT images. The OCT image of tumor mass compared with normal tissues was analyzed using its A-scan data so as to obtain a tissue attenuation rate which increases according to tumor growth.
\end{abstract}

Keywords: Optical coherence tomography, Tumor, Tissue attenuation, Mouse model

OCIS codes : (170.4500) Optical coherence tomography; (170.1470) Blood or tissue constituent monitoring; (170.6935) Tissue characterization; (170.0170) Medical optics and biotechnology

\section{INTRODUCTION}

Since optical coherence tomography (OCT) had been reported by the group of J. G. Fujimoto in 1991, many researchers have kept an eye on optical tomographic imaging by OCT due to its radiation-free high resolution imaging capability for tissues [1,2]. Radiation from medical imaging techniques such as X-ray computed tomography (CT) may induce cellular damage and increase the risk of cancer, although it is carefully controlled so as to keep annual radiation exposure low [3, 4]. Moreover, radiation-free tomographic imaging has importance in order to study radiation effectiveness on normal and tumor tissues. Although OCT is one of the radiation free and optical tomographic imaging techniques, it has very shallow imaging depth of a few millimeters and its usefulness is restricted. Therefore, OCT has been most useful in imaging in ophthalmology because of the transparency of ocular tissues [2, 5-9]. Recently, Ruggeri, et al. reported retinal tumor images of rodent in vivo and mentioned the difficulties of light delivery into the pupil of a small mouse eye $[10,11]$. Smith, et al. reported a molecular imaging method of early stage of cancer and blood vessels in auricle tissues of a nude mouse using quantum dots as an imaging agent [12]. Auricle tissues of BALB/c nude mice are promising for observing early stage of various cancers and can be easily aimed at for the therapy. Because the nude strain mouse lacks a thymus, it is relatively receptive to engrafted cells from foreign species. In addition, due to its hairlessness, it is easy to observe closely the progress of tumor formation after cancer cells have been inoculated under the skin [13, 14]. McLaughlin, et al. reported parametric OCT technique extracting tissue attenuation to reveal cancerous region from healthy tissues for human lymph nodes in vitro [15].

\footnotetext{
*Corresponding author: hwsong@etri.re.kr

Color versions of one or more of the figures in this paper are available online.
} 
In this paper, we report an optical monitoring method for tumor growth in auricle tissue of an animal model using optical coherence tomography in vivo. The cancer cells of PC-3 were cultured and inoculated into the hypodermis of auricle tissues in BALB/c nude mice to observe the tumor. Overall thickness of the auricle was observed as tomographic images in normal and tumor regions due to transparency of an ear of a $\mathrm{BALB} / \mathrm{c}$ nude mouse. The tomographic images of cancerous regions are compared with the images of normal tissues. Furthermore, optical attenuation estimation based on A-scan data analysis using high resolution OCT was studied for monitoring tumors in the early stage.

\section{EXPERIMENTS}

We took tomographic images of tumors in an animal model using a commercial spectral domain OCT (SD-OCT) system (OCP930SR, Thorlabs Inc. US). We used a superluminescent diode (SLD) as a light source at $950 \mathrm{~nm}$ wavelength to take high resolution tomographic images. Fig. 1 shows the schematic of the SD-OCT and the spectra of the SLD with the 3-dB bandwidth of $66 \mathrm{~nm}$. The SD-OCT
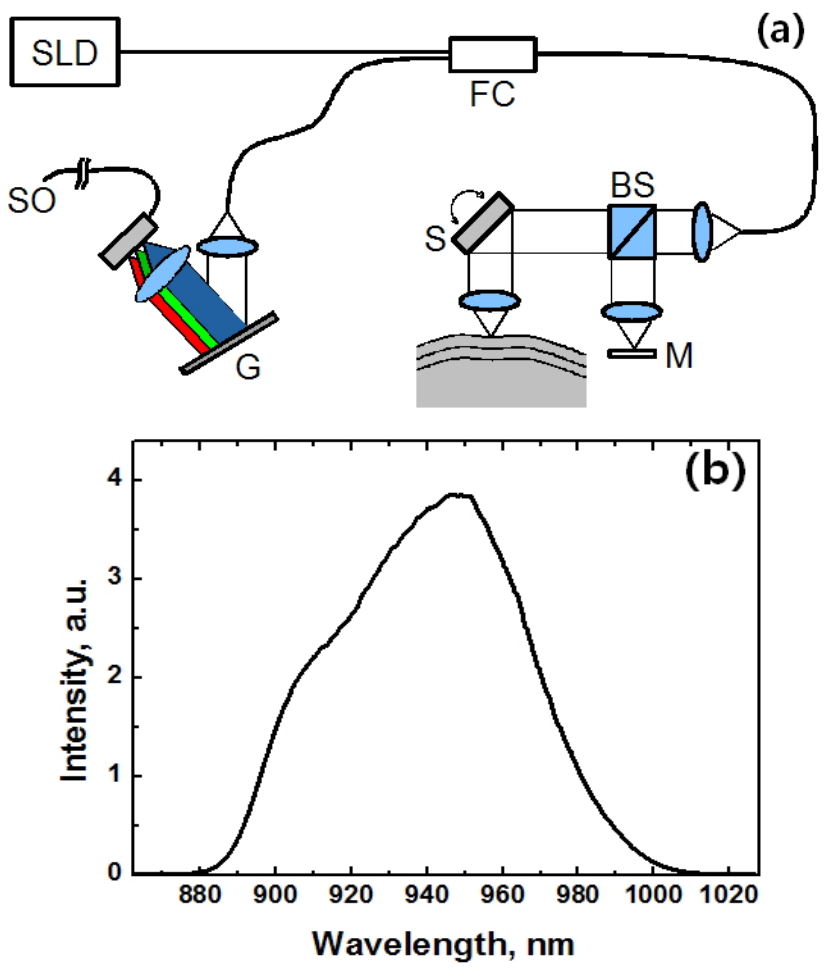

FIG. 1. Spectral domain optical coherence tomography (OCT) with the schematic as in (a) was used for optical tomographic imaging of tumors. The superluminescent diode (SLD) light source has spectra with center wavelength of 950 $\mathrm{nm}$ and 3-dB bandwidth of $66 \mathrm{~nm}$ (b). FC: fiber coupler, BS: beam splitter, G: grating, M: reference mirror, S: galvano scanner, SO: signal output. system consists of a beam splitter, a galvanometer scanner, a reference mirror, a fiber coupler, and a spectrometer. A-scan rate is approximately $5 \mathrm{kHz}$ with lateral scanning range up to $8 \mathrm{~mm}$ with image size of 512 rows so as to have B-scan rate of $8 \mathrm{fps}$. Axial resolution of $6.6 \mu \mathrm{m}$ is estimated by measuring the point spread function from a single reflective interface at $0.7 \mathrm{~mm}$ imaging depth as shown in Fig. 2. The SD-OCT system performances were measured to have sensitivity of $82.2 \mathrm{~dB}$ at $0.1 \mathrm{~mm}$ deep position from the focal plane and sensitivity roll-off rate of $-48.9 \mathrm{~dB} / \mathrm{mm}$. Sample attenuation of $29.6 \mathrm{~dB}$ was used for measuring sensitivities at various imaging depths.

We prepared the $\mathrm{BALB} / \mathrm{c}$ nude mouse as an animal model for imaging a solid tumor. Three or more mice were prepared as the age of around 4 weeks and anesthetized with conventional avertin (Sigma) intraperitoneal injection of approximately $0.4 \mathrm{~mL}$ for in vivo experiments. All experiments were performed in according to the regulation of Korea Atomic Energy Research Institute Animal Care and Use
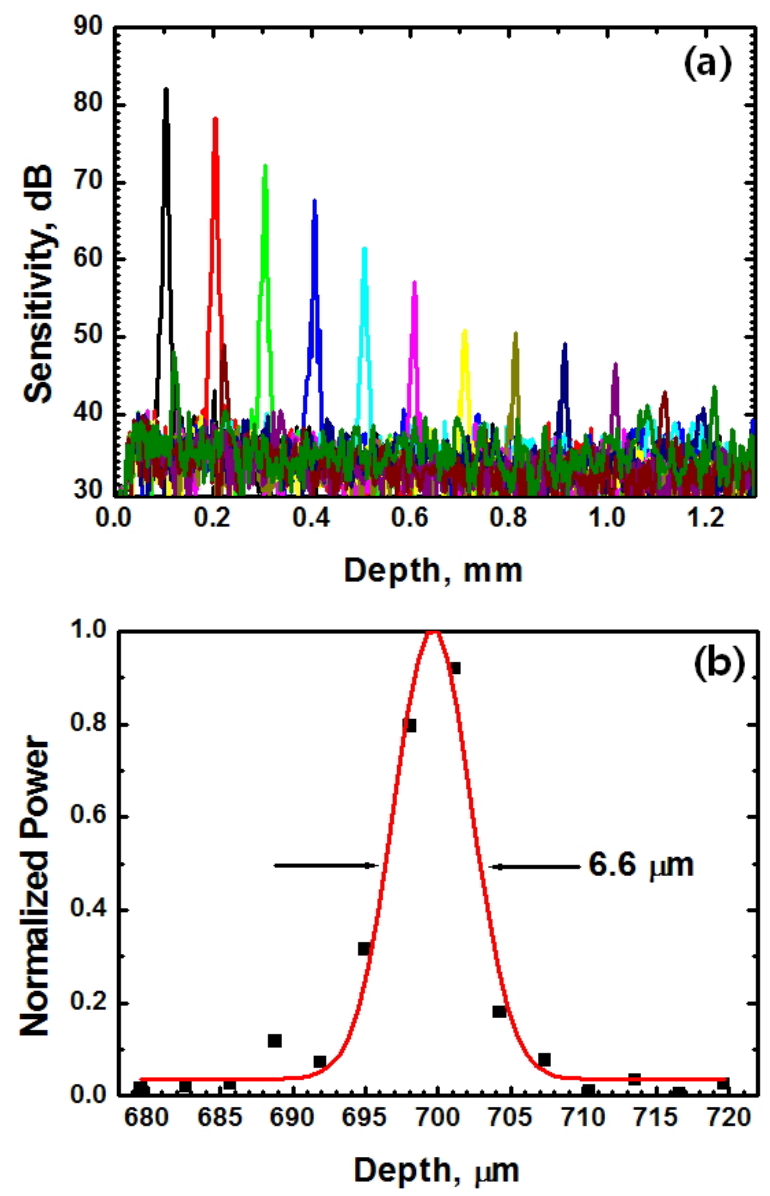

FIG. 2. Spectral-domain OCT system performance was measured as the sensitivity of $82.2 \mathrm{~dB}$ at $0.1 \mathrm{~mm}$ deep position from a focal plane and the sensitivity roll-off rate of -48.9 $\mathrm{dB} / \mathrm{mm}$ (a). Point spread function at $0.7 \mathrm{~mm}$ imaging depth shows an axial resolution of $6.6 \mathrm{~mm}$ (b). Sample attenuation of $29.6 \mathrm{~dB}$ was used for measuring sensitivities at various imaging depths. 


\section{Committee.}

The cancer cells of A-549 and PC-3 were cultured and subcutaneously injected into auricle tissues in BALB/c nude mice to generate the tumor. A-549 cells, adenocarcinomic human alveolar basal epithelial cells, are widely used as an in vitro model for a type II pulmonary epithelial cell and as a transfection host. PC-3 cells are known as classical cell lines of human prostatic cancer. When culturing A-549 and PC-3 adherent to the culture flask in vitro, we used traditional RPMI 1640 media with a standard antibiotic cocktail to prevent bacterial infection (streptomycin in combination with penicillin $1 \%$ and HyClone, Fetal Bovine Serum $10 \%$ ) and the environment of a $5 \%$ carbon dioxide at $37^{\circ} \mathrm{C}$. A-549 and PC-3 cells have been trypsinized and separated in order to obtain cancer cells in a solution. Nude mice were inoculated with 0.2 million cancer cells in low volume $(0.02 \mathrm{~mL})$ by gently pulling the skin of the ear and carefully sliding a 30 gauge needle through the skin a few $\mathrm{mm}$ [12]. We have used $95 \%$ glycerin on an auricle surface to reduce the strong reflection at the interface between the skin and air.

\section{RESULTS AND DISCUSSION}

Due to thinness and transparency of an ear of a BALB/c nude mouse, an in vivo cross sectional image was recorded throughout the overall thickness of normal auricle by SD-OCT as shown in Fig. 3. Tissue layers such as epidermis, dermis, and cartilage are clearly shown in an image obtained (Fig. 3(a)) and a magnified image (Fig. 3(b)). Embedded blood vessels that have been seen in the CCD images (lower Fig. 3(a)) are also shown as indicated with blue circles. A-scan data recorded across a blood vessel (along the red line) show local maxima of the power signals at the interfaces between tissue layers and air as shown in Fig. 3(c). The signal level in blood vessel was found out to be $3.9 \mathrm{~dB}$ lower than the minimum signal level in the other tissue regions. The area within blood vessel appears dark, while blood is flowing through the vessel in vivo. This effect is due to flowing blood that shifts the interference fringe frequency away from the phase modulation frequency [16-18]. All OCT images were recorded 5 times and shown as averaged images in order to cut down the effect of speckle noise. The point spread function measured at the interface between cartilage and dermis represents the axial resolution of $6.8 \mu \mathrm{m}$ that is slightly deteriorated by dispersion of tissue media (Fig. 3(d)).

We observed tumor formation and growth around an injection region of PC-3 cells and obtained the images of tumor mass center and sparse circumferences. Similar results were obtained in the experiments with the cancer cells of A-549 as well. The tomographic images of cancerous regions were compared with the images of normal tissues as in Fig. 4. OCT images of an auricle region in BALB/c nude mice were taken in vivo before and after a subcutaneous injection of cancer cells. Normal auricle has the overall optical thickness of about $350 \mu \mathrm{m}$ in Fig. 3(a). Swollen (a)
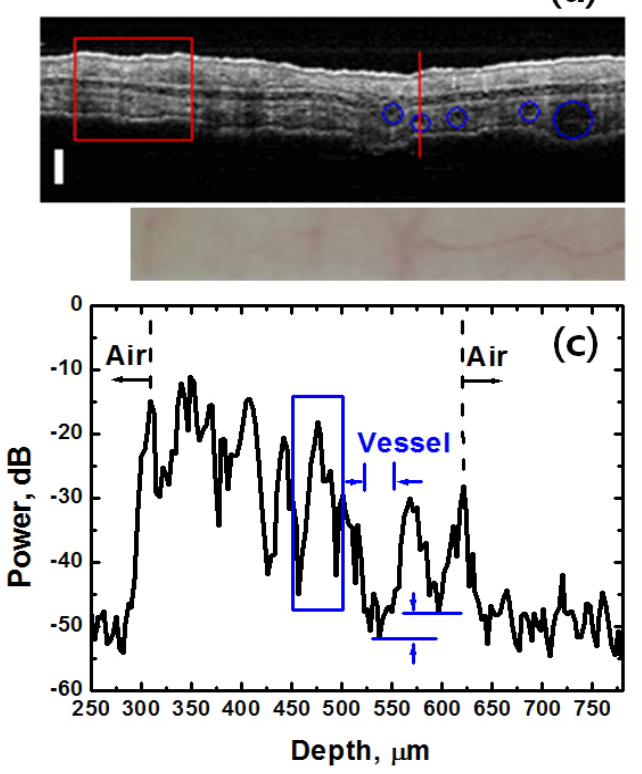

(b)
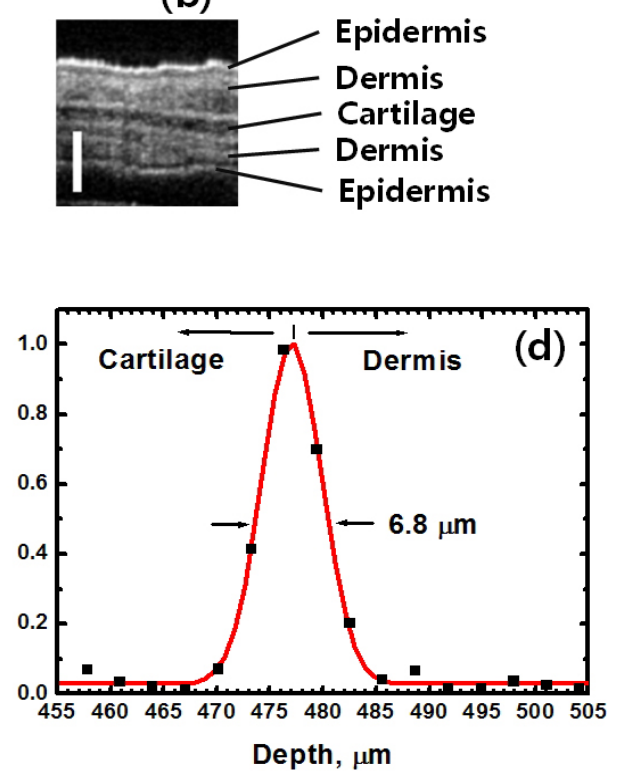

FIG. 3. An in vivo cross sectional image was recorded throughout overall thickness of normal auricle in BALB/c nude mice by spectral domain OCT (a). Tissue layers such as epidermis, dermis, and cartilage are clearly shown in the magnified image (b) of the red rectangle. A-scan data obtained along the red line show the power levels at each tissue layer and in air (c). The signal level in blood vessel was found to be $3.9 \mathrm{~dB}$ lower than the minimum signal level in the other tissue regions. The point spread function (d) represents the axial resolution of $6.8 \mu \mathrm{m}$ which was measured at the interface between cartilage and dermis in the blue rectangle. The blue circles include blood vessels as in CCD images (lower (a)). Scale bar: $200 \mu \mathrm{m}$. 

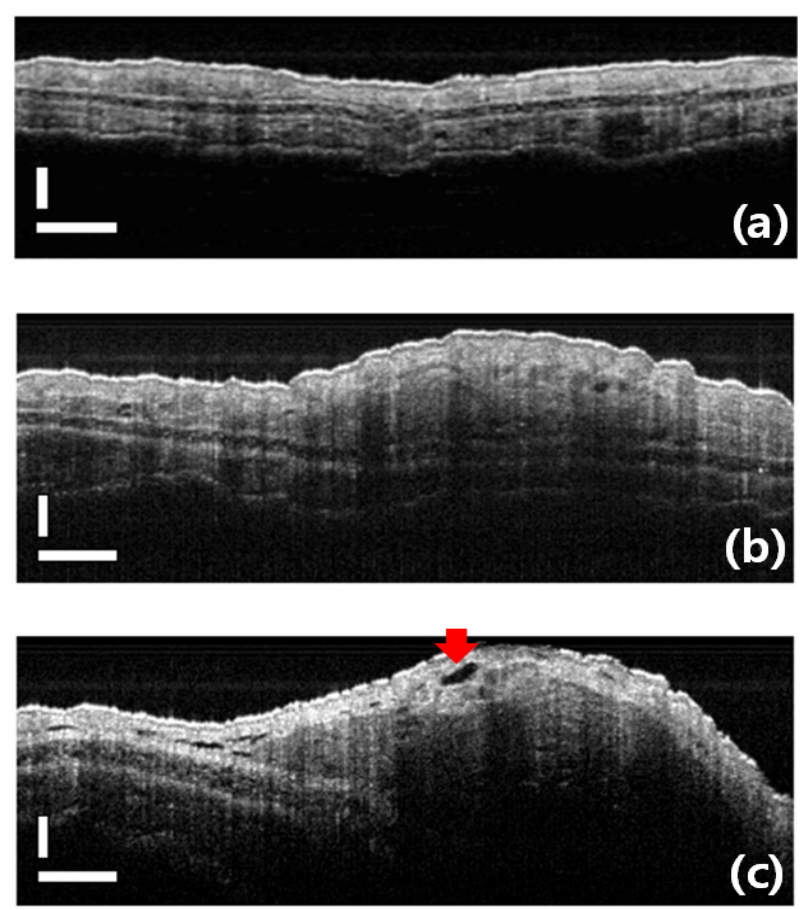

FIG. 4. OCT images of an auricle region in BALB/c nude mice were taken in vivo before and after an injection of cancer cells into the hypodermis. Normal auricle tissues have the overall optical thickness of about $350 \mu \mathrm{m}$ in the image (a). Swollen auricle tissues are shown in tomographic image (b) and (c) on the $3^{\text {rd }}$ and $5^{\text {th }}$ days from an inoculation, respectively. The red arrow points out the gap of significant size on which actively growing tumor mass borders. Scale bar: $200 \mu \mathrm{m}$ (vertical), $400 \mu \mathrm{m}$ (horizontal).
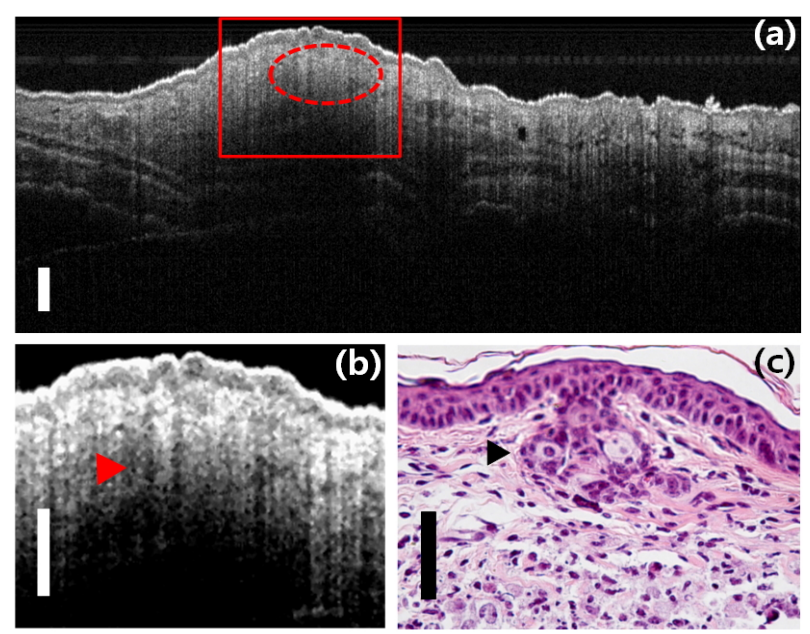

FIG. 5. An OCT image of the tumor region is shown on the $5^{\text {th }}$ day from an injection of cancer cells (a). The magnified image (b) of the red rectangle and the histological image (c) are arranged side by side so as to compare with each other. Tumor mass region is indicated with the dashed red line and pointed out by the red and black triangles. Blood vessels are shown around the tumor region as dark areas that have the lowest signal levels in the A-scan data of the tissue region. Scale bar: $200 \mu \mathrm{m}$. auricles are shown in the images on $3^{\text {rd }}$ and $5^{\text {th }}$ day from an injection, respectively (Figs. 4(b) and (c)). Tumor mass center and sparse circumferences around an injection region are shown as well. The tumor associated endothelial cells overlap one another and, as pointed by the red arrow, show a gap of significant size between them. Such a gap permits the seepage of plasma fluids into the interstitial spaces between the cancer cells, contributing to high hydrostatic pressure in these spaces [19].

Fig. 5(a) shows an OCT image of the cancerous region on the $5^{\text {th }}$ day from an inoculation of PC-3 cells. The magnified image of the red rectangle and the histological image are arranged side by side for comparison with each other. Tumor mass region is indicated with a dashed red line and pointed out by red and black arrows in Figs. 5(b) and (c), respectively. On the $5^{\text {th }}$ day from an inoculation, histological images of tumor regions using cross-sectional slicing and hematoxylin \& eosin (H\&E) staining of specimens were taken in order to confirm the correlation with the high resolution OCT images taken previously.

The leakiness of tumor-associated microvasculature results in the continuous leaking of thrombin and fibrinogen molecules from the plasma into the parenchyma surrounding blood vessels. Tissue factor is a protein displayed on the surface of cancer cells and many other cell types. Thrombin from the plasma is activated upon contact with tissue factor and converts fibrinogen into fibrin. This may result in the extensive network of fibrin bundles seen as an oval interface (indicated by red and black arrows) at the border between actively growing cancer cells and the tumor stroma (shown in Figs. 5(b) and (c)). A typical behavior of invasive carcinoma cells is shown as a clear interface, in which a well organized cohort (i.e. an invasive front) of these cells invades near to the stroma [19].

In general, the proliferation of the endothelial cells is encouraged by other cells in both the epithelium and stroma in order to guarantee access by all of these cells to an adequate blood supply. Especially in cancer formation, many cells lack adequate access to oxygen and release angiogenic factors actively that stimulate the ingrowth of capillaries [20]. Fig. 5(b) has many dark spots and considerable difference in comparison with the image of normal tissues in Fig. 3(b). It shows the generation of many blood capillaries around the tumor mass as dark spots on the image that have the lowest signal level in A-scan data of tissue images as mentioned previously (Fig. 3(c)). Blood capillaries appearing as dark spots in OCT images could be much larger than the imaging resolution of $6.8 \mu \mathrm{m}$ in their diameters. Lymph capillaries are slightly larger than their counterpart capillaries of the vascular system. However, it is known that lymphatic ducts are present in the normal tissue outside the tumor margin but are absent within the tumor mass itself [19]. Many blood capillaries were confirmed as many small holes in a histological image of the same region (Fig. 5(c)).

In order to quantify the overall amount of blood capillaries 

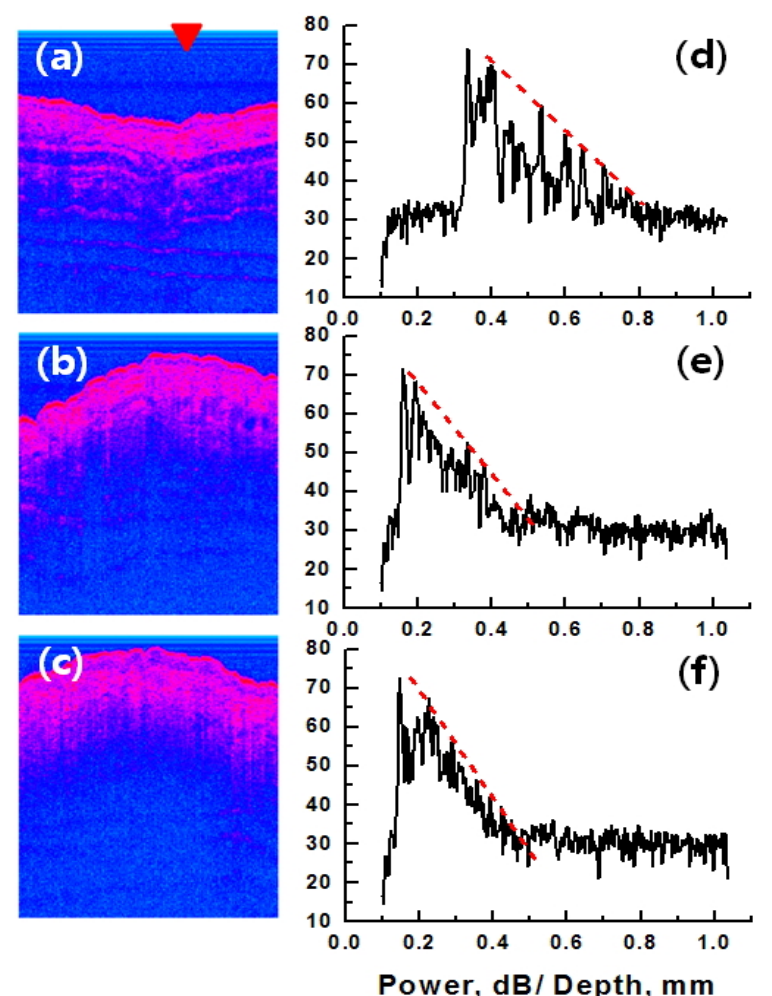

FIG. 6. OCT images and A-scan data on $1^{\text {st }}$ (before inoculation), $3^{\text {rd }}$ and $5^{\text {th }}$ days are shown in (a)-(d), (b)-(e) and (c)-(f), respectively. The A-scan data were brought from the OCT images at the position pointed out by the red triangle. The tomographic images and the A-scan data show that the tumor region has a strong rate of light attenuation compared to the normal tissues. Light attenuation is represented as a slope of the dashed red line that was linearly fitted to local maxima of signals from tissue interfaces.

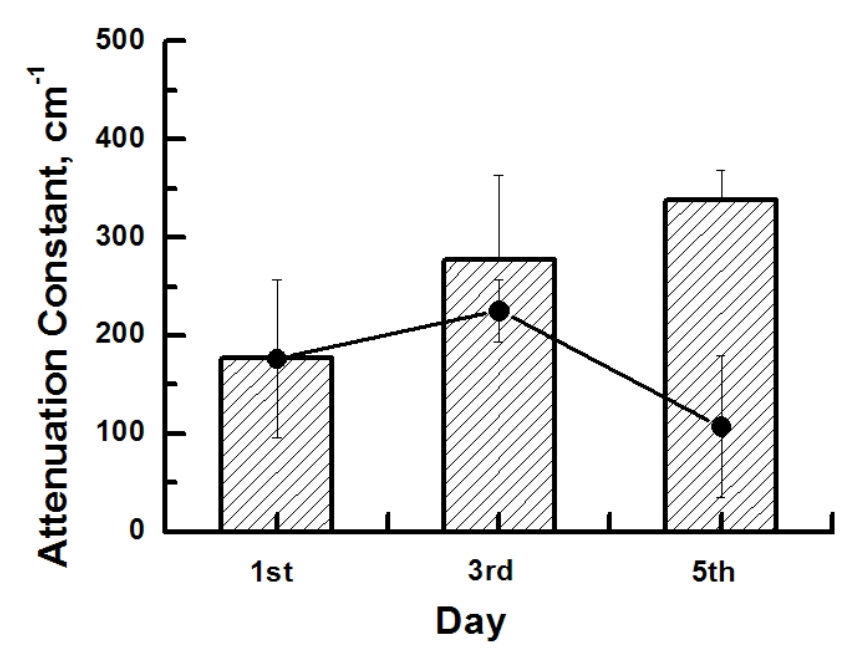

FIG. 7. Attenuation constants of the normal tissue region are compared with those of the tumor tissue region as a function of day in a histogram. Filled circles represent averaged attenuation constants of the normal tissue region in the same image on the day. A tumor has more optical attenuation rate by a few hundred $\mathrm{cm}^{-1}$ than the surrounding normal tissues due to its dense vasculature. around the tumor mass, OCT images were analyzed in the aspect of light attenuation. OCT images and their A-scan data on the $1^{\text {st }}$ (before inoculation), $3^{\text {rd }}$ and $5^{\text {th }}$ days are shown in Figs. 6(a)-(d), (b)-(e) and (c)-(f), respectively. The A-scan data were brought from the recorded OCT images of Figs. 6(a), (b) and (c) at the position pointed to by the red triangle. The light attenuation constant is proportional to a slope of the dashed red line that was linearly fitted to local maxima of signal appearing from tissue interfaces. The steeper the slope of the A-scan signal, the larger the optical attenuation constant in the measured tissues. The tumor region had a strong rate of light attenuation compared with the normal tissues as in Figs. 6(d), (e) and (f).

We estimated attenuation constants of the tissues by subtracting the sensitivity roll-off (i.e. instrumental roll-off) from the slopes of A-scan data and considering light path length as double that of the tissue thickness. Averaged attenuation constants of the normal tissue region are compared with those of the tumor tissue region in the histogram of Fig. 7. Filled circles represent averaged attenuation constants of the normal tissue region in the same OCT image on each day. On the $3^{\text {rd }}$ day, the attenuation constant of the tumor tissues was $101 \mathrm{~cm}^{-1}$ larger than that of the same region before inoculation and $52 \mathrm{~cm}^{-1}$ larger than that of the surrounding normal tissues on the same day. The attenuation constant on the $5^{\text {th }}$ day was $162 \mathrm{~cm}^{-1}$ and 231 $\mathrm{cm}^{-1}$ larger than those of the normal tissues in the same and surrounding regions, respectively. Optical attenuation in skin is mainly attributed to melanin absorption in the epidermis and blood scattering in the dermis. The epidermal attenuation constant, which is considerably variable according to melanin content, is about $80 \mathrm{~cm}^{-1}$ at $950 \mathrm{~nm}$ where the melanin content is a $10 \%$ volume fraction of melanosomes [21]. The effect of the epidermal layer on tissue attenuation is relatively restricted because of its thinness. The attenuation constant in whole blood, mainly due to light scattering by red blood cells, is known to be as large as about $670 \mathrm{~cm}^{-1}$ at $960 \mathrm{~nm}[22,23]$. Therefore, due to dense vasculature, tumor tissues had more optical attenuation by a few hundred $\mathrm{cm}^{-1}$ than the normal tissues. Moreover, optical attenuation rate increase according to tumor growth is mainly attributed to angiogenesis in the tumor region.

\section{CONCLUSION}

In conclusion, OCT images of tumor mass in an animal model were investigated using SD-OCT having the axial resolution of $6.8 \mu \mathrm{m}$ at the center wavelength of $950 \mathrm{~nm}$. The cancer cells of PC-3 were cultured and inoculated into auricle tissues in BALB/c nude mice to observe the tumor in vivo. We observed tumor formation at an injection region of cancer cells and obtained the images of tumor mass center and sparse circumferences. The OCT images of cancer regions were compared with the images of normal tissues. Overall thickness of the auricle was observed as tomographic 
images in normal and tumor regions in $\mathrm{BALB} / \mathrm{c}$ nude mice. On the $5^{\text {th }}$ day after injection of cancer cells, histological images of tumor regions were taken in order to confirm the correlation with the high resolution OCT images. Tumor mass had a clear oval interface at the border between actively growing cancer cells and the tumor stroma. The interface could be the extensive network of fibrin bundles. It was remarkably different in comparison with normal tissues that the tumor image had many blood capillaries around the tumor mass showing as dark spots. That was explained by the signal level in A-scan data of the blood vessels and the histological images. Moreover, a tumor had more optical attenuation by a few hundred $\mathrm{cm}^{-1}$ than the normal tissues due to its dense vasculature. Tissue attenuation estimation based on high resolution OCT images could be useful for monitoring tumors in the early stage.

\section{ACKNOWLEDGMENT}

This work was supported by the ETRI support program (100356020) and the Industrial Strategic Technology Development Program (10042581) funded by the Ministry of Knowledge Economy, Korea.

\section{REFERENCES}

1. D. Huang, E. A. Swanson, C. P. Lin, J. S. Schuman, W. G. Stinson, W. Chang, M. R. Hee, T. Flotte, K. Gregory, C. A. Puliafito, and J. G. Fujimoto, "Optical coherence tomography," Science 254, 1178-1181 (1991).

2. W. Drexler, U. Morgner, R. K. Ghanta, F. X. Kärtner, J. S. Schuman, and J. G. Fujimoto, "Ultrahigh resolution ophthalmic optical coherence tomography," Nature Medicine 7, $502-507$ (2001).

3. J. L. Marti, L. T. Dauer, M. Stempel, S. Patil, J. B. Kaplan, and L. L. Montgomery, "Cumulative imaging radiation exposure following breast-conservation therapy," Annals of Surgical Oncology 18, 104-108 (2011).

4. H. Jeong, J.-E. Rah, U.-J. Hwang, S. H. Yoo, B. J. Min, S.-Y. Lee, M. Yoon, D. H. Shin, S. Y. Park, S. B. Lee, and J.-Y. Kim, "Estimation of the secondary cancer risk induced by diagnostic imaging radiation during proton therapy," Journal of Radiological Protection 31, 477-487 (2011).

5. A. F. Fercher, C. K. Hitzenberger, W. Drexler, G. Kamp, and H. Sattmann, "In vivo optical coherence tomography," American Journal of Ophthalmology 116, 113-114 (1993).

6. A. A. Moayed, S. Hariri, E. S. Song, V. Choh, and K. Bizheva, "In vivo volumetric imaging of chicken retina with ultrahigh-resolution spectral domain optical coherence tomography," Biomedical Optics Express 2, 1268-1274 (2011).

7. S.-W. Lee, H.-W. Song, M.-Y. Jung, and S. Kim, "Wide tuning range wavelength-swept laser with a single SOA at $1020 \mathrm{~nm}$ for ultrahigh resolution Fourier-domain optical coherence tomography," Opt. Express 19, $21227-21237$ (2011).

8. S.-W. Lee, H.-W. Song, B.-K. Kim, M.-Y. Jung, S.-H. Kim, J. D. Cho, and C.-S. Kim, "Fourier domain optical coherence tomography for retinal imaging with $800 \mathrm{~nm}$ swept source: real time resampling in k-domain," J. Opt. Soc. Korea 15, 293-299 (2011).

9. T. E. J. Fabritius, S. Makita, M. Yamanari, R. A. Myllyla, and Y. Yasuno, "Complex conjugate resolved retinal imaging by one-micrometer spectral domain optical coherence tomography using an electro-optical phase modulator," J. Opt. Soc. Korea 15, 111-117 (2011).

10. M. Ruggeri, H. Wehbe, S. Jiao, G. Gregori, M. E. Jockoich, A. Hackam, Y. Duan, and C. A. Puliafito, "In vivo three dimensional high resolution imaging of rodent retina with spectral domain optical coherence tomography," Investigative Ophthalmology \& Visual Science 48, 1808-1814 (2007).

11. M. Ruggeri, G. Tsechpenakies, S. Jiao, M. E. Jockoich, C. Cebulla, E. Hernandez, T. G. Murray, and C. A. Puliafito, "Retinal tumor imaging and volume quantification in mouse model using spectral domain optical coherence tomography," Opt. Express 17, 4074-4083 (2009).

12. B. R. Smith, Z. Cheng, A. De, J. Rosenberg, and S. S. Gambhir, "Dynamic visualization of RGD quantum dot binding to tumor neovasculature and extravasation in multiple living mouse models using intravital microscopy," Small 6, 22222229 (2010).

13. J.-T. Oh, S.-W. Lee, Y.-S. Kim, K.-B. Suhr, and B.-M. Kim, "Quantification of the wound healing using polarizationsensitive optical coherence tomography," Journal of Biomedical Optics 11, 041124 (2006).

14. T. Zhang, J.-L. Li, X.-C. Ma, J. Xin, and Z.-H. Tu, "Reliability of phototoxic tests of fluoroquinolones in vitro," Acta Pharmacological Sinica 24, 453-459 (2003).

15. R. A. McLaughlin, L. Scolaro, P. Robbins, C. Saunders, S. L. Jacques, and D. D. Sampson, "Parametric imaging of cancer with optical coherence tomography," Journal of Biomedical Optics 15, 046029-1 046029-4 (2010).

16. Z. Chen, T. E. Milner, D. Dave, and J. S. Nelson, "Optical Doppler tomographic imaging of fluid flow velocity in highly scattering media," Opt. Lett. 22, 64-66 (1997).

17. Z. Chen, T. E. Milner, S. Srinivas, X. Wang, A. Malekafzali, M. J. C. van Gemert, and J. S. Nelson, "Noninvasive imaging of in vivo blood flow velocity using optical Doppler tomography," Opt. Lett. 22, 1119-1121 (1997).

18. Z. Chen, Y. Zhao, S. M. Srinivas, J. S. Nelson, N. Prakash, and R. D. Frostig, "Optical Doppler tomography," IEEE J. Select. Topics Quantum Electron. 5, 1134-1142 (1999).

19. R. A. Weinberg, The Biology of Cancer (Garland Science, NY, USA, 2007).

20. J. Folkman, "Angiogenesis in cancer, vascular, rheumatoid and other disease," Nature Medicine 1, 27-31 (1995).

21. S. L. Jacques and D. J. McAuliffe, "The melanosome: threshold temperature for explosive vaporization and internal absorption coefficient during pulsed laser irradiation," Photochemistry and Photobiology 53, 769-775 (1991).

22. W. F. Cheong, S. A. Prahl, and A. J. Welch, "A review of the optical properties of biological tissues," IEEE J. Quantum Electron. 26, 2166-2175 (1990).

23. M. Meinke, G. Muller, J. Helfmann, and M. Friebel, "Empirical model functions to calculate hematocrit-dependent optical properties of human blood," Appl. Opt. 46, 1742-1753 (2007). 